\title{
Experimental investigation of the asymmetric spectroscopic characteristics of electron- and hole-doped cuprates
}

\author{
N.-C. Yeh, ${ }^{a *}$ C.-T. Chen, ${ }^{a}$ A. D. Beyer, ${ }^{a}$ C. R. Hughes, ${ }^{a}$ T. A. Corcovilos, ${ }^{a}$ S. I. Lee ${ }^{b}$ \\ ${ }^{a}$ Department of Physics, California Institute of Technology, Pasadena, CA 91125, USA \\ ${ }^{b}$ Department of Physics, Pohang University of Science and Technology,Pohang,Korea
}

\begin{abstract}
Quasiparticle tunneling spectroscopic studies of electron- (n-type) and hole-doped (p-type) cuprates reveal that the pairing symmetry, pseudogap phenomenon and spatial homogeneity of the superconducting order parameter are all non-universal. We compare our studies of p-type $\mathrm{YBa}_{2} \mathrm{Cu}_{3} \mathrm{O}_{7-\delta}$ and n-type infinite-layer $\mathrm{Sr}_{0.9} \mathrm{Ln}_{0.1} \mathrm{CuO}_{2}(\mathrm{Ln}=\mathrm{La}, \mathrm{Gd})$ systems with results from p-type $\mathrm{Bi}_{2} \mathrm{Sr}_{2} \mathrm{CaCu}_{2} \mathrm{O}_{\mathrm{x}}$ and n-type one-layer $\mathrm{Nd}_{1.85} \mathrm{Ce}_{0.15} \mathrm{CuO}_{4}$ cuprates, and attribute various non-universal behavior to different competing orders in p-type and n-type cuprates.
\end{abstract}

Keywords: Quasiparticle spectra; pseudogap; pairing symmetry; competing orders

\section{Introduction}

The presence of competing orders in the ground state of cuprate superconductors [1] results in rich phenomena and complications for unraveling the pairing mechanism. Recent experimental development [1-3] reveals significant non-universal phenomena and asymmetric characteristics between n-type and p-type cuprates as the consequences of competing orders. In particular, the asymmetric characteristics among n-type and p-type cuprates may be attributed to the differences in their low-energy spin excitations $[1,2]$. We suggest that the incommensurate spin excitations associated with charge modulations in p-type cuprates may result in a charge nematic $(\mathrm{CN})$ phase that competes with superconductivity (SC), yielding pseudogap phenomena and nano-scale phase separations in twodimensional (2D) cuprates and long-range SC order in 3D cuprates $[1,2]$. In contrast, commensurate spin excitations in $n$-type cuprates, as manifested by neutron scattering [4] and implied by quasiparticle spectra [2,3], are indicative of the coexistence of antiferromagnetism (AFM) with SC.

\section{Competing orders and pseudogap in p-type cuprates}

The doping of holes into the $\mathrm{CuO}_{2}$ planes of cuprates is known to induce gapped spin excitations in the $\mathrm{CuO}_{2}$ plane. One of the possible ground states of the hole-doped $\mathrm{CuO}_{2}$ plane is the stripe phase which accommodates gapped spin excitations via charge modulations [1]. However, a charge stripe phase with long-range order is energetically very costly. A compromised competing ground state phase in the presence of disorder could be a $\mathrm{CN}$ phase that involves local charge modulations while evading strong Coulomb repulsion. In general, the competition between two order parameters can result in three possible phase diagrams as a function of the chemical potential [1-3]: 1) nano-scale phase separations of the two phases, 2) coexistence of the two phases, and 3) disorder intermediate between the two phases. For highly 2D p-type cuprates such as $\mathrm{Bi}_{2} \mathrm{Sr}_{2} \mathrm{CaCu}_{2} \mathrm{O}_{\mathrm{x}}(\mathrm{Bi}-2212)$, the resulting ground state could reflect either Case 1) or Case 2), because SC involving continuous $\mathrm{U}(1)$ symmetry breaking cannot sustain longrange homogeneity in 2D [2], and also because the $\mathrm{CN}$ phase could be better stabilized by local disorder in 2D [8].

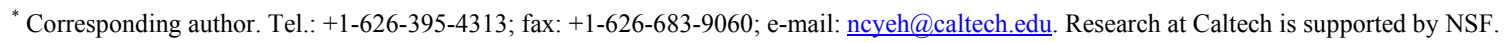


The $\mathrm{CN}$ phase may extend above $T_{c}$ and yield the pseudogap (PG) phenomenon. In contrast, highly 3D p-type cuprates like $\mathrm{YBa}_{2} \mathrm{Cu}_{2} \mathrm{O}_{7-\delta}$ (YBCO) may belong to Case 2), with homogeneous $\mathrm{SC}$ manifested by scanning tunneling spectroscopy [1], NMR [5] and microwave [1] studies. Figure 1 compares the spatial evolution of quasiparticle

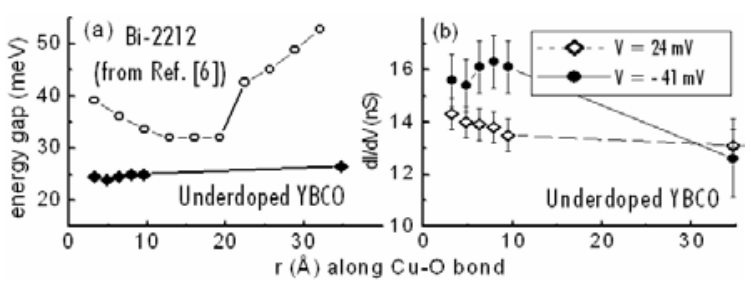

spectral characteristics between YBCO and Bi-2212.

Fig.1 Spatial evolution of quasiparticle spectral features along the $\mathrm{Cu}-\mathrm{O}$ bonding direction: (a) energy gap of underdoped YBCO $\left(T_{c} \approx 60 \mathrm{~K}\right)[1]$ and $\mathrm{Bi}-2212\left(T_{c} \approx 79 \mathrm{~K}\right)$ [6]; (b) differential conductance $(\mathrm{dI} / \mathrm{dV})$ of underdoped YBCO at bias voltages $\mathrm{V}=24 \mathrm{mV}$ and $-41 \mathrm{mV}$.

To investigate the possibility of locally pinned $\mathrm{CN}$ coexisting with SC in Bi-2212, we consider the effects of three sources of elastic scattering on the energy $(E)$ and momentum transfer $(\boldsymbol{q})$ dependence of the tunneling conductance $\Delta G(\boldsymbol{q}, E)$, which is proportional to the Fouriertransformed local density of states (FT-LDOS) $\rho_{q}(E)[7,8]$ : point defects in the $\mathrm{SC}$ region, stripes in the pinned $\mathrm{CN}$ region, and 1D "edge states" separating the $\mathrm{CN}$ and $\mathrm{SC}$ regions. Assuming weak scattering potentials and using the first-order T-matrix approximation [8], we find that $\rho_{q}(E) \propto$ $\int d^{3} k \delta\left(E-E_{k}\right) \delta\left(E-E_{k+q}\right)|V(\boldsymbol{q})| F_{ \pm}(k, \boldsymbol{q})$, where $|V(\boldsymbol{q})|$ is the elastic scattering matrix in the Born approximation, and $F_{ \pm}(k, \boldsymbol{q})$ are the coherence factors for spin-independent $(+)$ and spin-dependent (-) interactions [8]. Examples of the quasiparticle FT-LDOS are shown in Figs. 2(a)-(c), where we have included 24 randomly distributed defects in an area of $\left(200 a_{0} \times 200 a_{0}\right)$. We note that the FT-LDOS in Fig. 2(b) due to $\mathrm{CN}$ scattering of quasiparticles reveals more intense features along the $(\pi, 0) /(0, \pi)$ directions than those due to point and edge defects in Figs. 2(a) and 2(c). The combined spectra of both point- and $1 \mathrm{D}$-scattering centers seem to agree better with experimental observation $[7,8]$, implying that pinned $\mathrm{CN}$ could coexist with $\mathrm{SC}$ in $\mathrm{Bi}-2212$.

\section{Coexisting AFM and SC in n-type cuprates}

The presence of commensurate spin excitations in onelayer n-type cuprates, as revealed from neutron scattering data [4], is consistent with the coexistence of a long-range AFM order parameter in the SC state, and the absence of charge modulations may account for the absence of PG above $T_{c}$ [9]. Further evidence for coexisting AFM and SC is provided by our quasiparticle spectra of the n-type infinite-layer cuprate $\mathrm{Sr}_{0.9} \mathrm{La}_{0.1} \mathrm{CuO}_{2}$ : For small tunneling currents $(<20 \mathrm{nA})$, similar spectral characteristics with $\Delta_{S C}$ $=13 \mathrm{meV}$ prevail, whereas for large tunneling currents $(>$ $80 \mathrm{nA})$, a different large-gap spectrum $\left(\Delta_{A F M} \sim 25 \mathrm{meV}\right)$ emerges. The field-induced pseudogap below $T_{c}$ and above the upper critical field $B_{c 2}(T)$ for one-layer n-type cuprates [9] is also suggestive of remnant AFM or a spin-flop (SF) phase upon the suppression of $\mathrm{SC}$ at $B>B_{c 2}(T)$. In Fig. 3 we compare the phase diagram of the n-type infinite-layer (from our magnetization measurements) with that of the one-layer cuprates [9]. The different vortex phase boundaries of $\mathrm{Sr}_{0.9} \mathrm{Gd}_{0.1} \mathrm{CuO}_{2}$ and $\mathrm{Sr}_{0.9} \mathrm{La}_{0.1} \mathrm{CuO}_{2}$ suggest excess pinning effects due to the magnetic moments of $\mathrm{Gd}$.

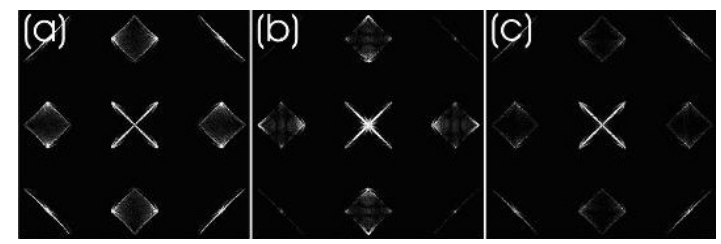

Fig.2 Quasiparticle FT-LDOS associated with (a) nonmagnetic point defects, (b) stripes in $\mathrm{CN}$, and (c) edge states for $\left(q_{x}, q_{y}\right)=\left( \pm \pi / a_{0}, \pm \pi / a_{0}\right), \Delta_{d}=40 \mathrm{meV}$ and $E=20$ $\mathrm{meV}$. The sharper contrast represents stronger interference intensity.
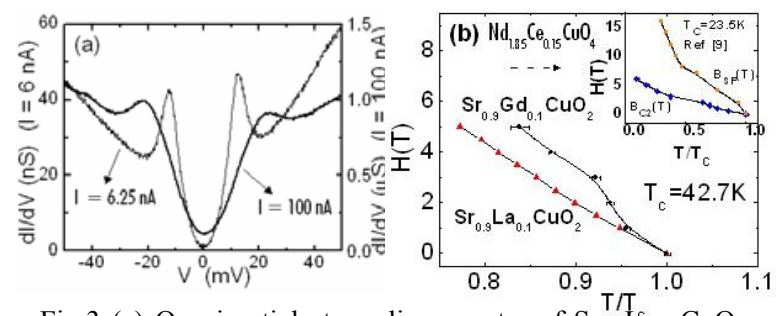

Fig.3 (a) Quasiparticle tunneling spectra of $\mathrm{Sr}_{0.9}^{\mathrm{T} / \mathrm{La}_{0.1}} \mathrm{CuO}_{2}$ at $4.2 \mathrm{~K}$ taken with tunneling currents of $I=6.25 \mathrm{nA}$ and $100 \mathrm{nA}$. (b) Magnetic phase diagram of n-type cuprates: infinite-layer (main panel) and one-layer (inset). In the inset $\mathrm{B}_{\mathrm{SF}}(\mathrm{T})$ refers to a spin-flop phase for $\mathrm{H} \| \mathrm{c}$-axis.

\section{References}

[1] N.-C. Yeh, Bulletin of Assoc. Asia Pacific Phys. Soc. Vol. 12, No. 2 (2002) 2 [cond-mat/0210656].

[2] N.-C. Yeh and C.-T. Chen, Int. J. Mod. Phys. B 17 (2003) 3575 [cond-mat/0302217].

[3] C.-T. Chen et al., Phys. Rev. Lett. 88 (2002) 227002.

[4] M. Matsuda et al., Phys. Rev. B 66 (2002) 172509.

[5] J. Bobroff et al., Phys. Rev. Lett. 89 (2002) 157002.

[6] K. M. Lang et al. Nature 415 (2002) 412.

[7] J. E. Hoffman et al., Science 297 (2002) 1148.

[8] C.-T. Chen and N.-C. Yeh, Phys. Rev. B 68 (2003) 220505(R) [cond-mat/0307660].

[9] S. Kleefisch et al., Phys. Rev. B 63 (2001) 100507. 